\title{
Vertex Corrections and the Korringa Ratio in Strongly Correlated Electron Materials
}

\author{
Eddy Yusuf ${ }^{1,2}$, B. J. Powell ${ }^{2}$, and Ross H. McKenzie ${ }^{2}$ \\ ${ }^{1}$ Department of Physics, University at Buffalo, SUNY, Buffalo, New York 14260-1500, USA \\ ${ }^{2}$ Centre for Organic Photonics and Electronics, School of Physical Sciences, \\ University of Queensland, Brisbane, Queensland 4072, Australia
}

(Dated: September 5, 2021)

\begin{abstract}
We show that the Korringa ratio, associated with nuclear magnetic resonance in metals, is unity if vertex corrections to the dynamic spin susceptibility are negligible, the hyperfine coupling is momentum independent, and there exists an energy scale below which the density of states is constant. In the absence of vertex corrections we also find a Korringa behaviour for $T_{1}$, the nuclear spin relaxation rate, i.e., $1 / T_{1} \propto T$, and a temperature independent Knight shift. These results are independent of the form and magnitude of the self-energy (so far as is consistent with neglecting vertex corrections) and of the dimensionality of the system.
\end{abstract}

Nuclear magnetic resonance (NMR) spectroscopy is a powerful experimental probe of the spin dynamics of strongly correlated electron materials. An important quantity in NMR experiments on metals is the Korringa ratio,, $.2 \mathcal{K}$, which is proportional to the ratio of the $\mathrm{nu}-$ clear relaxation rate $1 / T_{1}$ to the square of the Knight shift, $K_{s}$. The Korringa ratio, and the temperature dependence of $1 / T_{1}$ and $K_{s}$ can provide important insights into the electronic and magnetic correlations. In this paper we show that, under certain conditions, the Korringa ratio does not deviate from its non-interacting value even in strongly correlated electron systems.

In the diagrammatic formalism of quantum many-body theory, the effects of electronic correlations are described by the self energy and the vertex corrections. The self energy describes the effect that interactions with virtual particles have on the propagation of particles through the material. Vertex corrections describe the renormalisation of coupling constants due to interactions (the name arises because coupling constants appear at the vertices in Feynman diagrams) $\underline{\underline{3}}$

An important consideration in the study of vertex corrections is Migdal's theorem, which states that the vertex corrections due to the electron-phonon interaction are of order $\sqrt{m / M}$, where $m$ is the electron mass and $M$ is the nuclear mass. The Eliashberg theory of superconductivity, which improves on BCS theory by replacing the BCS effective pairwise interaction with an explicit treatment of the electron-phonon interaction, invokes Migdal's theorem in order to neglect vertex corrections. There is no Migdal's theorem for either the electron-electron interaction or the electron-magnon interaction $\stackrel{4}{ }$ Therefore, understanding the importance of vertex corrections in strongly correlated superconductors is of great importance and has been widely debated. Hertz et al. $\frac{5}{n}$ have shown that the first order vertex corrections due to paramagnons is the same order of magnitude as the bare vertex. More recently, in the context of the cuprates, arguments have been presented both for $\frac{6.7 .8}{.6}$ and against $\underline{9.10,11}$ the thesis that vertex corrections are negligible. Some theories of strongly correlated superconductors have at- tempted to deal with the vertex corrections. For example, for the one band Hubbard model, the FLEX approximation $\frac{12}{12}$ consists of a self-consistent summation of bubble and ladder diagrams; the latter are the lowest order self-constient contributions to the vertex function.

The relaxation of the nuclei is governed by their coupling to their environment, which, in a metal, is the conduction electrons $\frac{1.2}{2}$ Hence, many of the properties measured in NMR experiments depend on the transverse dynamic magnetic susceptibility of the electron fluid, $\chi_{-+}\left(\mathbf{q}, i \omega_{n}\right)$. In Matsubara formalism, this is given by 13

$$
\chi_{-+}\left(\mathbf{q}, i \omega_{n}\right)=\int_{0}^{\beta} d \tau e^{i \omega_{n} \tau}\left\langle T_{\tau} m_{-}(\mathbf{q}, \tau) m_{+}(-\mathbf{q}, 0)\right\rangle,
$$

where $\beta=1 / k_{B} T$ is the inverse temperature, $\tau$ is the imaginary time, $\omega_{n}$ are the Matsubara frequencies,

$$
\begin{aligned}
& m_{-}(\mathbf{q}, \tau)=\frac{\hbar \gamma_{e}}{\sqrt{2}} \sum_{\mathbf{p}} c_{\mathbf{p}+\mathbf{q}, \downarrow}^{\dagger}(\tau) c_{\mathbf{p}, \uparrow}(\tau), \\
& m_{+}(\mathbf{q}, \tau)=\frac{\hbar \gamma_{e}}{\sqrt{2}} \sum_{\mathbf{p}} c_{\mathbf{p}+\mathbf{q}, \uparrow}^{\dagger}(\tau) c_{\mathbf{p}, \downarrow}(\tau),
\end{aligned}
$$

are the $\mp$ components of magnetization, and $T_{\tau}$ is the (imaginary) time ordering operator.

The Korringa ratio $\frac{1.2}{2}$ is the dimensionless quantity

$$
\mathcal{K} \equiv \frac{\hbar}{4 \pi k_{B}}\left(\frac{\gamma_{e}}{\gamma_{N}}\right)^{2} \frac{1}{T_{1} T K_{s}^{2}}
$$

where $\gamma_{N}\left(\gamma_{e}\right)$ is the nuclear (electronic) gyromagnetic ratio. The Korringa ratio is unity in a non-interacting system with a contact, i.e. momentum independent, hyperfine coupling. The hyperfine coupling is momentum independent if there is one atom per unit cell, and this is an approximation otherwise $\stackrel{2}{2}$ Further, Korringa showed that in such a system $1 / T_{1} T$ and $K_{s}$ are independent

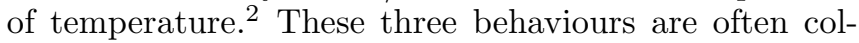
lectively referred to as Korringa behaviour. However, real materials always exhibit some correlations and the Korringa ratio may deviate from unity, or $1 / T_{1} T$ or $K_{s}$ 
may be temperature dependent $14,15,16$ In elemental metals the Korringa ratio is typically between 0.6 and 1 (see Ref. 1, pp. 156-7). Further, the Korringa ratio is greater (less) than unity if the system is near an antiferromagnetic (ferromagnetic) instability $\stackrel{17}{ }$ There are large deviations from Korringa behaviour in a wide range of strongly correlated electron materials including the cuprates, 18 organic charge transfer salts,,$\frac{19,20}{2}$ and the heavy fermion materials. 21 On the other hand, many materials and model Hamiltonians do show Korringa behaviour; including some strongly correlated ones, such as magnetic impurities described by the Anderson model ${ }^{22}$ Therefore it is important to determine what conditions may be sufficient for Korringa behaviour in a correlated system.

The relaxation rate and the Knight shift can be written in terms of the dynamic spin susceptibility,

$$
\begin{aligned}
\frac{1}{T_{1} T} & =\lim _{\omega \rightarrow 0} \frac{2 k_{B}}{\gamma_{e}^{2} \hbar^{4}} \sum_{\mathbf{q}}|A(\mathbf{q})|^{2} \frac{\chi_{-+}^{\prime \prime}(\mathbf{q}, \omega)}{\omega} \\
K_{s} & =\frac{|A(\mathbf{0})| \chi_{-+}^{\prime}(\mathbf{0}, 0)}{\gamma_{e} \gamma_{N} \hbar^{2}}
\end{aligned}
$$

where $A(\mathbf{q})$ is the hyperfine coupling between the nuclear and electron spins, and $\chi^{\prime}(\mathbf{q}, \omega)\left[\chi^{\prime \prime}(\mathbf{q}, \omega)\right]$ is the real [imaginary] part of the dynamic susceptibility.

The focus of this paper is on how electronic correlations affect the Korringa ratio. Specifically, we investigate how vertex corrections modify the Korringa ratio and what implications this has for strongly correlated materials. We show that as zero temperature is approached the Korringa ratio approaches unity if three conditions are satisfied: (i) vertex corrections are negligible, (ii) the hyperfine coupling is momentum independent, and (iii) there exits a energy scale below which the density of states is constant. This result holds independent of the strength of the electron-electron interactions that enter the self energy and of the dimensionality of the system. We also find that, under the same conditions, $1 / T_{1} T$ and $K_{s}$ are independent of temperature. Hence, non-Korringa behaviour must result from vertex corrections, a momentum dependent hyperfine coupling and/or the absence of an energy scale below which the density of states is constant.

Upon substituting (2) into (11), performing the appropriate Wick contractions on the operators, and Fourier transforming into frequency space one finds that

$$
\begin{aligned}
\chi_{-+}\left(\mathbf{q}, i \omega_{n}\right)= & \frac{\hbar^{2} \gamma_{e}^{2}}{2 \beta} \sum_{\mathbf{p}, i p_{m}} \Gamma\left(\mathbf{p}+\mathbf{q}, i p_{m} ; \mathbf{p}, i p_{m}+i \omega_{n}\right) \\
& \times G\left(\mathbf{p}+\mathbf{q}, i p_{m}\right) G\left(\mathbf{p}, i p_{m}+i \omega_{n}\right),
\end{aligned}
$$

where $\Gamma\left(\mathbf{q}, i \omega_{n} ; \mathbf{p}, i \omega_{n}^{\prime}\right)$ [shaded area] is the three point vertex function and $G\left(\mathbf{p}, i p_{n}\right)$ [solid lines] is the full in- teracting Green's function given, in the spectral representation, by

$$
G\left(\mathbf{p}, i p_{n}\right)=\int_{-\infty}^{\infty} \frac{d E_{1}}{2 \pi} \frac{A_{s}\left(\mathbf{p}, E_{1}\right)}{i p_{n}-E_{1}},
$$

where $A_{s}\left(\mathbf{p}, E_{1}\right)$ is the spectral function, given by

$$
A_{s}(\mathbf{p}, E)=\frac{-2 \operatorname{Im} \Sigma(\mathbf{p}, E)}{\left(E-\varepsilon_{\mathbf{p}}-\operatorname{Re} \Sigma(\mathbf{p}, E)\right)^{2}+(\operatorname{Im} \Sigma(\mathbf{p}, E))^{2}},
$$

$\varepsilon_{\mathbf{p}}$ is the dispersion of the non-interacting system, and $\Sigma\left(\mathbf{p}, i p_{n}\right)$ is the self energy. Therefore

$$
\begin{aligned}
\chi_{-+}\left(\mathbf{q}, i \omega_{n}\right)= & \frac{\hbar^{2} \gamma_{e}^{2}}{2 \beta} \sum_{\mathbf{p}, m} \int_{-\infty}^{\infty} \frac{d E_{1}}{2 \pi} \frac{d E_{2}}{2 \pi} \\
& \times \Gamma\left(\mathbf{p}+\mathbf{q}, i p_{m} ; \mathbf{p}, i p_{m}+i \omega_{n}\right) \\
& \times \frac{A_{s}\left(\mathbf{p}+\mathbf{q}, E_{1}\right) A_{s}\left(\mathbf{p}, E_{2}\right)}{\left(i p_{m}-E_{1}\right)\left(i p_{m}+i \omega_{n}-E_{2}\right)} .
\end{aligned}
$$

At this stage we neglect vertex corrections, that is we set $\Gamma\left(\mathbf{q}, i \omega_{n} ; \mathbf{p}, i p_{n}\right)=1$ for all $\mathbf{p}, \mathbf{q}, p_{n}$, and $\omega_{n}$. After performing the Matsubara summation and the analytical continuation $i \omega_{n} \rightarrow \omega+i \eta$, one finds that

$$
\begin{aligned}
\chi_{-+}(\mathbf{q}, \omega)= & \frac{\hbar^{2} \gamma_{e}^{2}}{2} \sum_{\mathbf{p}} \int_{-\infty}^{\infty} \frac{d E_{1}}{2 \pi} \frac{d E_{2}}{2 \pi} A_{s}\left(\mathbf{p}+\mathbf{q}, E_{1}\right) \\
& \times A_{s}\left(\mathbf{p}, E_{2}\right) \frac{n_{F}\left(E_{1}\right)-n_{F}\left(E_{2}\right)}{\hbar \omega+E_{1}-E_{2}+i \eta}
\end{aligned}
$$

where $n_{F}(E)$ is the Fermi function.

Using the well known equality $1 /(x+i \eta)=\mathcal{P}(1 / x)-$ $i \pi \delta(x)$, where $\mathcal{P}(y)$ denotes the principal value, one finds

$$
\begin{array}{r}
\lim _{\omega \rightarrow 0} \frac{\chi_{-+}^{\prime \prime}(\mathbf{q}, \omega)}{\hbar \omega}=\frac{\hbar^{2} \gamma_{e}^{2}}{2} \sum_{\mathbf{p}} \int_{-\infty}^{\infty} \frac{d E}{4 \pi} A_{s}(\mathbf{p}+\mathbf{q}, E) \\
\times A_{s}(\mathbf{p}, E)\left(-\frac{\partial n_{F}}{\partial E}\right) .
\end{array}
$$

It then follows from Eq. (4a) that

$$
\frac{1}{T_{1} T}=\frac{k_{B}|A|^{2}}{\hbar} \int_{-\infty}^{\infty} \frac{d E}{4 \pi} \tilde{\rho}^{2}(E)\left(-\frac{\partial n_{F}}{\partial E}\right),
$$

where $\tilde{\rho}(E)=\sum_{\mathbf{p}} A_{s}(\mathbf{p}, E)$ is the full interacting density of states per spin species and we have assumed a contact hyperfine coupling, $A(\mathbf{q})=A$ for all $\mathbf{q}$. We now specialise to the case where their exists an energy scale, $k_{B} T_{0}$ below which $\tilde{\rho}(E)$ is independent of energy. There several situations in which $T_{0}$ may not exist or may be too small to be of practical interest, for example, if the Fermi energy is at, or very close to, a van Hove singularity or if there is a (pseudo)gap at the Fermi energy. For $T \ll T_{0}, T_{F}$, where $T_{F}$ is the Fermi temperature, Eq. (12) simplifies to

$$
\frac{1}{T_{1} T} \simeq \frac{k_{B}|A|^{2} \tilde{\rho}^{2}\left(E_{F}\right)}{4 \pi \hbar}
$$


Note that the right hand side, and therefore $1 / T_{1} T$, is independent of temperature.

For $H \rightarrow 0$, where $H$ is a static magnetic field, $\chi_{-+}^{\prime}(\mathbf{0}, 0)=\chi_{z z}^{\prime}(\mathbf{0}, 0)=\partial M /\left.\partial H\right|_{H=0}$, where $\chi_{z z}^{\prime}(\mathbf{q}, \omega)$ is the real part of the longitudinal component of the dynamic spin susceptibility and the magnetisation, $M$ is given by $\underline{23}$

$$
M=\frac{\hbar \gamma_{e}}{2} \sum_{\mathbf{k}}\left(\left\langle\hat{n}_{\mathbf{k} \uparrow}\right\rangle-\left\langle\hat{n}_{\mathbf{k} \downarrow}\right\rangle\right),
$$

where $\hat{n}_{\mathbf{k} \sigma}$ is the usual number operator. Writing $\hat{n}_{\mathbf{k} \sigma}$ in the spectral representation and performing the sum over $\mathrm{k}$ one finds that

$$
M=\frac{\hbar \gamma_{e}}{2} \sum_{\sigma} \sigma \int_{-\infty}^{\infty} \frac{d E}{2 \pi} \tilde{\rho}_{\sigma}(\mathbf{k}, E) n_{F \sigma}(E)
$$

where we take $\sigma= \pm 1, \rho_{\sigma}(E)=\sum_{\mathbf{k}} A_{s \sigma}(\mathbf{k}, E)$ is the density of states of spin $\sigma$ electrons and $A_{s \sigma}(\mathbf{k}, E)$ is the spectral function for spin $\sigma$ electrons. Even for small magnetic fields the Fermi surface may, in general, be distorted $^{23}$ as $E_{\mathbf{k} \sigma}=E_{\mathbf{k}}-\sigma H \Gamma\left(\mathbf{k}, E_{\mathbf{k} \sigma} ; \mathbf{k}, E_{\mathbf{k} \sigma}\right) \hbar \gamma_{e} / 2+$ $\mathcal{O}\left(H^{2}\right)$. However, in the absence of vertex corrections such complications cannot arise as the $\mathbf{k}$-dependence drops out of the above equation. Therefore we deal with the magnetic field by introducing a spin dependent chemical potential: $\mu_{\sigma}=\mu-\sigma H \hbar \gamma_{e} / 2+\mathcal{O}\left(H^{2}\right)$ and noting that $n_{F \sigma}=\left\{1+\exp \beta\left[\left(E-\mu_{\sigma}\right)\right]\right\}^{-1}$. Thus one finds that,

$$
\lim _{T \rightarrow 0} M=\frac{\hbar \gamma_{e}}{2} \sum_{\mathbf{k} \sigma} \sigma \int_{-\infty}^{\infty} \frac{d E}{2 \pi}\left[\tilde{\rho}_{\sigma}(E) \Theta\left(E-\mu_{\sigma}\right)+\sigma \frac{\hbar \gamma_{e}}{2} \tilde{\rho}_{\sigma}(E) \delta\left(E-\mu_{\sigma}\right) H+\frac{\partial \tilde{\rho}_{\sigma}(E)}{\partial H} \Theta\left(E-\mu_{\sigma}\right)+\mathcal{O}\left(H^{2}\right)\right] .
$$

In the limit $H \rightarrow 0$ the first term vanishes due to spin symmetry and the third term vanishes from the assumption that we are at an energy scale on which the density of states is constant. Thus, the Knight shift is

$$
K_{s} \simeq \frac{|A| \gamma_{e} \tilde{\rho}\left(E_{F}\right)}{4 \pi \gamma_{N}}
$$

which is independent of temperature.

It follows immediately from Eqs. (13) and (17), that $\mathcal{K}=1$ for interacting electrons with a contact hyperfine coupling when $T \ll T_{0}, T_{F}$ and neglecting vertex corrections. We stress two points about this result: firstly, our result includes the special case of Korringa's result for the free electron gas, $\mathcal{K}_{\text {free }}=1$; secondly, and more importantly, the Korringa ratio is unity for a broad class of systems and not just for the free electron gas.

Thus we have shown that any deviation of the Korringa ratio from unity, or temperature dependence of $1 / T_{1} T$ or $K_{s}$ must be caused by either vertex corrections, the wavevector dependence of the hyperfine coupling, or the fact the there is no energy, $k_{B} T_{0}$, on which we may treat the density of states as constant. Note that this result is independent of the dimensionality of the system. Further, this is true for any form of the self energy. However, it should be noted that the self-energy and the vertex function are not really independent: they both arise from the same underlying interactions and can often be related by Ward identities. So this last statement should be taken with appropriate caution.

Let us now briefly discuss the role of vertex corrections in strongly correlated systems. A simple and a widely studied approach to nearly (anti)ferromagetic metals is the random phase approximation (RPA). For the Hubbard model the RPA gives, $\frac{13}{,}$

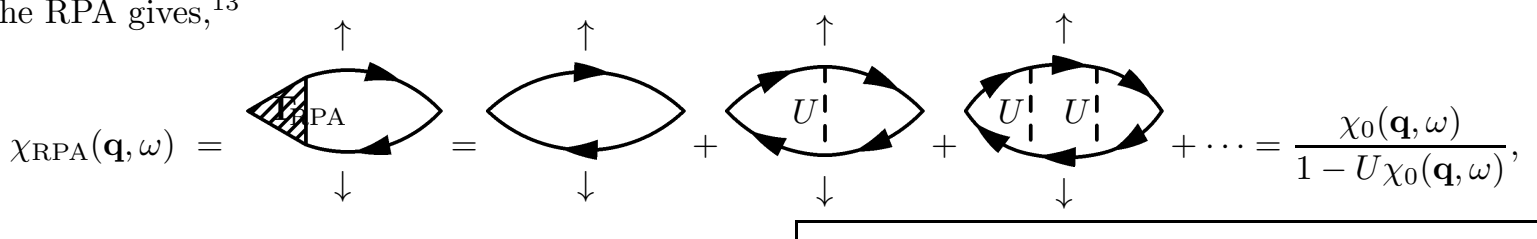

where $U$ (dashed lines) is the effective Coulomb interaction between electrons on the same lattice site, $\chi_{0}$ is the dynamic susceptibility in the absence of vertex corrections, and we have suppressed momentum labels in the diagrams for clarity. The distinctive 'Stoner-like' form of Eq. (18) arises from the sum of ladder diagrams, i.e., vertex corrections. For this form of $\chi(\mathbf{q}, \omega)$ and a three- dimensional parabolic band the Korringa ratio is always less than unity and decreases monotonically towards zero as the Stoner instability is approached $i^{15}$ However, for a two-dimensional parabolic band the free electron spin susceptibility is momentum independent. Consequently, c.f. Eqs. (4), the Korringa ratio remains unity for all $U$ even though $1 / T_{1}$ and the Knight shift both diverge as 
the Stoner instability is approached ${ }^{24}$

Korringa type relations have been derived for the impurity spin susceptibility, $\chi_{i m p}(\omega)$, of the Anderson single impurity model. It is found that 22,25

$$
\lim _{\omega \rightarrow 0} \frac{\chi_{i m p}^{\prime \prime}(\omega)}{\omega}=\frac{2 \pi \chi_{i m p}^{\prime}(0)^{2}}{\gamma_{e}^{2}}
$$

which is sometimes referred to as the Shiba relation. This relation holds even though there can be significant vertex corrections for the spin susceptibility. This and the case of the two-dimensional RPA with a parabolic band illustrate that the absence of vertex corrections is sufficient but not necessary for the Korringa ratio to be unity.

A similar form of $\chi(\mathbf{q}, \omega)$ to that given in (18) is found in dynamical mean field theory (DMFT), but with $U$ replaced by a self-consistently determined four-point vertex function. ${ }^{26}$ In DMFT, as in the RPA, this functional form results from a sum over ladder diagrams, with the four-point vertex function now forming the legs of the ladders. DMFT for the Hubbard model is equivalent to an Anderson single impurity model which is solved self-consistently. ${ }^{26}$ It follows that the local spin susceptibility $\chi_{l o c}(\omega)=\sum_{\mathbf{q}} \chi(\mathbf{q}, \omega)$ must satisfy the Shiba relation (19) with $\chi_{i m p}(\omega)=\chi_{l o c}(\omega)$. Since, in general, $\chi_{l o c}(\omega) \neq \chi(\mathbf{q}=0, \omega)$ the Korringa relation does not necessarily hold in DMFT [see Eq. (73) in Ref. 26].

A slave-boson large $N$ treatment of the periodic Anderson model shows that in the low-temperature heavy fermion phase the Korringa ratio is close to unity, even though $1 / T_{1} T$ and $K_{s}$ can be several orders of magnitude larger than the value predicted in the absence of strong electronic correlations. 27

In the singlet superconducting states the opening of the gap causes a suppression of both $1 / T_{1} T$ and $K_{s}$. For a fully gapped superconductor both $1 / T_{1} T$ and $K_{s}$ show activated behaviours, while if the order parameter has nodes power laws are seen at low $T .28$ The behaviour, particularly of the Knight shift, is more com- plicated in a triplet superconductor ${ }^{28}$ In the same way, a (pseudo)gap destroys the energy scale $T_{0}$ and hence causes non-Korringa behaviour by suppressing $1 / T_{1} T$ and $K_{s}$.

Given the above discussion it is interesting to consider the normal states of a few superconductors which display non-Korringa behaviour. A strong temperature dependence and a Korringa ratio significantly larger than unity is observed in the organic charge transfer salts, $, 19,20$ e.g., in $\kappa$-(ET) $)_{2} \mathrm{Cu}\left[\mathrm{N}(\mathrm{CN})_{2}\right] \mathrm{Br}$ (Refs. 29, 30). Heavy fermion compounds 31 and cobaltates 32 also show enhanced Korringa ratios above their Kondo temperatures. Most recently non-Korringa behaviour has been observed in the iron pnictides. ${ }^{33}$ In the cuprates, the Korringa ratio of $\mathrm{YBa}_{2} \mathrm{Cu}_{3} \mathrm{O}_{6.64}$ (Refs. 34, 35) has a strong temperature dependences and is larger than unity. However, in the cuprates the hyperfine coupling has a significant wave vector dependence. 36,37 This complicates deducing the relative importance of vertex corrections. We also note that vertex corrections are required in the superconducting state in order to preserve gauge invariance.

Moriya's self-consistent renormalised theory ${ }^{14}$ and the phenomenological MMP theory ${ }^{18}$ give a good description

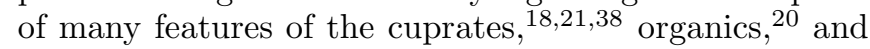
heavy fermion materials,$\stackrel{21}{2}$. These theories posit a form of $\chi(\mathbf{q}, \omega)$ which follows from the form of Eq. (18). Hence, these theories implicitly include vertex corrections.

In summary, we have shown that for a system with a contact hyperfine coupling the Korringa ratio is unity in the absence of vertex corrections provided there is an energy scale on which the density of states may be treated as constant. At sufficiently low temperatures $1 / T_{1} T$ and the Knight shift are both independent of temperature under the same assumptions.

This work was supported by the Australian Research Council (ARC) under the Discovery scheme (project DP0557532). BJP was the recipient of an ARC Queen Elizabeth II Fellowship (DP0878523). RHM was the recipient of an ARC Professorial Fellowship (DP0877875).
1 C. P. Slichter, Principles of Magnetic Resonance (Springer, Berlin, 1996), 2nd ed.

2 J. Korringa, Physica 16, 601 (1950).

${ }^{3}$ For an accessible discussion of these concepts see R. D. Mattuck, A Guide to Feynman diagrams in the Many-Body Problem (Dover, New York, 1976).

4 Some care needs to be taken when discussing the role off vertex corrections in effective low-energy Hamiltonians. It is important to make clear which Hamiltonian one is considering, as the vertex corrections in the original Hamiltonian may be integrated out of the effective Hamiltonian. For example, when studying the spin-fluctuation theory of superconductivity ${ }^{39}$ one starts from a Hubbard model and integrates out the high energy degrees of freedom, including those responsible for magnetism, so as be be left with an effective Hamiltonian containing both spins and fermions. Thus in the effective Hamiltonian $m_{ \pm}(\mathbf{q}, \tau)$ is not given by Eqs. (2) as it depends on the spins as well as the fermions. Thus, in this paper, when we use the term "vertex corrections" we are referring to models for which Eqs. (2) hold, unless we specify otherwise.

5 J. A. Hertz, K. Levin, and M. T. Beal-Monod, Solid State Commun. 18, 803 (1976).

${ }^{6}$ U. Chatterjee et al, Phys. Rev. B 75, 172504 (2007).

7 P. B. Littlewood et al., Phys. Rev. B 48, 487 (1993).

8 C. M. Varma et al., Phys. Rev. Lett. 63, 1996 (1989).

9 A. J. Millis, Phys. Rev. B 45, 13047 (1992).

10 H.-B. Schüttler and M. Norman, Phys. Rev. B 54, 13295 (1996).

11 Y. Bang, Phys. Rev. B 60, 7458 (1999).

12 N. E. Bickers and D. J. Scalapino, Ann. Phys. 193, 206 (1989); P. Monthoux and D. J. Scalapino, Phys. Rev. Lett. 72, 1874 (1994); C.-H. Pao and N. E. Bickers, ibid. 72, 1870 (1994). 
13 See for example, S. Doniach and E. H. Sondheimer, Green's Functions for Solid State Physics (Imperial College Press, London, 1998), 2nd ed., chapter 7.

14 T. Moriya, J. Phys. Soc. Jpn. 18, 516 (1963).

15 A. Narath and H. T. Weaver, Phys. Rev. 175, 373 (1968).

16 B. S. Shastry and E. Abrahams, Phys. Rev. Lett. 72, 1933 (1994).

17 S. Doniach, J. Appl. Phys. 39, 483 (1968).

18 A. J. Millis, H. Monien, and D. Pines, Phys. Rev. B 42, 167 (1990).

19 B. J. Powell and R. H. McKenzie, J. Phys.: Condens. Matter 18, R827 (2006).

20 E. Yusuf, B. J. Powell, and R. H. McKenzie, Phys. Rev. B 75, 214515 (2007).

21 N. J. Curro et al., Nature 434, 622 (2005).

22 A. C. Hewson, The Kondo Problem to Heavy Fermions (Cambridge University Press, Cambridge, 2001).

23 J. M. Luttinger, Phys. Rev. 119, 1153 (1960).

${ }^{24}$ H. Nishihara, I. Shirotani, and N. Inoue, J. Phys. Soc. Jpn. 48, 1957 (1980).

25 A. Yoshimori and A. Zawadowski, J. Phys. C 15, 5241 (1982).
26 A. Georges et al., Rev. Mod. Phys. 68, 1 (1996).

27 S. Evans and B. Coqblin, Phys. Rev. B 43, 12790 (1991).

28 V. P. Mineev and K. V. Samokhin, Introduction to Unconventional Superconductivity (Gordan and Breach, Amsterdam, 1999).

29 S. M. De Soto et al., Phys. Rev. B 52, 10364 (1995).

${ }^{30}$ K. Miyagawa, A. Kawamoto, and K. Kanoda, Phys. Rev. Lett. 89, 017003 (2002).

31 N. Büttgen et al., Phys. Rev. B 62, 11545 (2000).

${ }^{32}$ C. de Vaulx et al., Phys. Rev. Lett. 98, 246402 (2007).

33 Y. Naka et al., arXiv:0804.4765. H. Fukazawa, et al., arXiv:0806.4514.

34 M. Takigawa et al., Phys. Rev. B 43, 247 (1991).

35 R. E. Walstedt et al., Phys. Rev. B 41, 9574 (1990).

${ }^{36}$ F. Mila and T. M. Rice, Phys. Rev. B 40, 11382 (1989).

37 B. S. Shastry, Phys. Rev. Lett. 63, 1288 (1989).

38 T. Moriya and K. Ueda, Adv. Phys. 49, 555 (2000).

39 See, e.g., A. V. Chubukov, D. Pines, J. Schmalian, in The Physics of Superconductors, K. H. Bennemann and J. B. Ketterson (Eds.) (Springer, Berlin, 2003); P. Monthoux, D. Pines, and G. G. Lonzarich, Nature 450, 1177 (2007). 